\title{
Research note: Calculating spectral irradiance indoors
}

\author{
S Bará $\mathrm{PhD}^{\mathrm{a}}$ and J Escofet $\mathrm{PhD}^{\mathrm{b}}$ \\ á́rea de Óptica, Facultade de Óptica e Optometría, Universidade de Santiago de \\ Compostela, Santiago de Compostela, Galicia, Spain. \\ ${ }^{b}$ Departament d'Òptica i Optometria, Universitat Politècnica de Catalunya, Terrassa, \\ Catalunya, Spain.
}

Short title: Spectral irradiance indoors

Received 28 July 2016; Revised 11 August 2016; Accepted 13 August 2016

The spectral composition of the light that reaches any indoor work plane depends on the characteristics of the light sources and the spectral reflectances of the surrounding surfaces due to the multiple reflections experienced by the light rays along their paths from the source to the observation point. We show that in indoor spaces the source and surface radiances must obey a definite self-consistent relationship derived from the fact that each illuminated surface point acts as a secondary source of light. It is then established that the spectral irradiance on any plane is linearly dependent on the spectral radiance of the light source. The explicit integral form of this relationship provides a theoretical framework for a quantitative description of the surface effects. Additionally, under very general assumptions, we show that the spectral irradiance can be computed from the spectral flux of the source through a simple multiplication by a wavelength-dependent function. This function, with units of inverse surface $\left(1 / \mathrm{m}^{2}\right)$, provides a convenient way for evaluating the effects that arbitrary changes in the source spectrum will produce on the spectral irradiance at the indoor point under study.

Address for correspondence: Salvador Bará, Área de Óptica, Facultade de Óptica e Optometría, Universidade de Santiago de Compostela. 15782 Santiago de Compostela, Galicia, Spain. E-mail: salva.bara@usc.es 


\section{Introduction}

In indoor settings the spectral composition of the light that actually reaches the point of interest no matter whether it be on a work plane or at the cornea of the eye depends on the light source spectrum and on the spectral reflectance of the surrounding surfaces ${ }^{1}$ Predicting the spectral irradiance at any arbitrarily oriented indoor surface requires quantitative models that incorporate the spectral reflectance of the surrounding surfaces. In this Research Note we show that in indoor spaces the source and surface radiances must obey a self-consistent relationship, from which the linear dependence of the spectral irradiance on the spectral radiance of the source can be formally established. Furthermore, and under very general assumptions, this linear relationship allows us to define an effective inverse surface function (EISF), with units $\mathrm{m}^{-2}$, that, once multiplied by the source spectral radiant flux $\left(\mathrm{W} \cdot \mathrm{nm}^{-1}\right)$, directly provides the spectral irradiance $\left(\mathrm{W} \cdot \mathrm{m}^{-2} \cdot \mathrm{nm}^{-1}\right)$ on the plane of interest. The EISF depends on the position and orientation of the measurement plane, on the characteristics of the environment, and on the radiance distribution of the source. It does not depend on the source spectrum. The EISF allows for a rapid evaluation of the effects that any change of the spectral composition of the light source may have on the observed spectral irradiance, keeping constant the remaining geometrical parameters.

\section{Light sources in indoor spaces}

The spectral irradiance on any arbitray indoor plane contains contributions from the direct radiance from the light source and the radiance reflected from the points of the environment acting as secondary sources. Since any point of the environment, in turn, 
is illuminated not only by the light source but also by the light reflected at the remaining points, the reflected radiances are mutually dependent on each other and have to obey the self-consistent relationship (See equation A5 of the Appendix).

Another way to consider this is to say that light arrives to the defined point following multiple paths. Some rays travel along a straight line connecting the source to the point without modifiying their spectral composition, neglecting for all practical purposes any differential absorption or scattering effects due to the air present within the room. Other rays, however, reach that plane after having undergone one or more diffuse reflections at the surrounding surfaces. Every time the light is diffusely reflected, its spectral composition is modified by the local bidirectional spectral reflectance distribution function (BSRDF). Consequently, the spectral composition of the light at the plane can be very different from the original spectrum of the source.

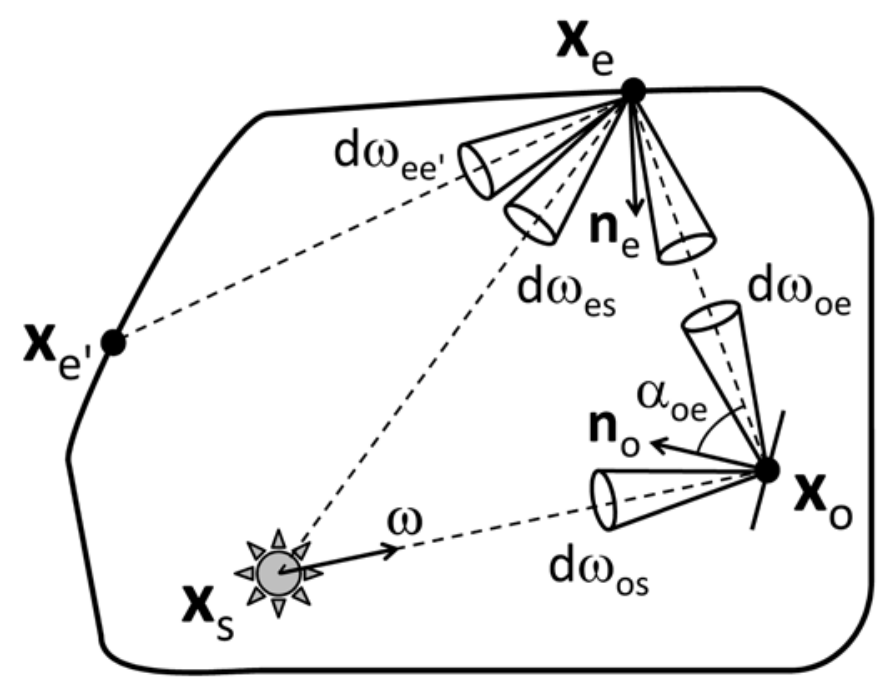

Figure 1. The spectral irradiance at a point $\mathbf{x}_{o}$ of the plane of interest, whose normal is described by the vector $\mathbf{n}_{o}$, is made up from the radiance directly received from the source (located at $\mathbf{x}_{s}$ ) and the radiance reflected from the points $\left(\mathbf{x}_{e}\right)$ of the surrounding surfaces. 
The spectral irradiance at any given point from multiple reflections can be mathematically described by a series of recursively nested integrals (see Appendix, equation A7), each one accounting for the light arriving at that point after a given number of diffuse reflections. This series can be straightforwardly deduced from the above quoted self-consistent relationship, as shown in the Appendix. As it could be anticipated, the final result shows that the irradiance at the plane of interest, $E_{O}\left(\lambda ; \mathbf{x}_{O}, \mathbf{n}_{O}\right)$, although spectrally different from the radiance arising from the light source, $L_{S}\left(\lambda ; \mathbf{x}_{S}, \boldsymbol{\omega}\right)$, depends linearly on it. This can be formally expressed as:

$$
E_{O}\left(\lambda ; \mathbf{x}_{O}, \mathbf{n}_{O}\right)=\ell_{\lambda}\left[L_{S}\left(\lambda ; \mathbf{x}_{S}, \boldsymbol{\omega}\right)\right]
$$

where $\ell_{\lambda}$ stands for linear operator (see equations A7 and A8 in the Appendix), $\lambda$ is the wavelength, $\mathbf{x}_{O}$ is the position vector of the point on the surface where the irradiance is evaluated, $\mathbf{n}_{O}$ is the unit vector perpendicular to the plane at that point, $\mathbf{x}_{S}$ is the position vector of the source, and $\boldsymbol{\omega}$ is a unit vector along the emission directions of the source, as shown in Figure 1. $E_{O}\left(\lambda ; \mathbf{x}_{O}, \mathbf{n}_{O}\right)$ is measured in $\mathrm{W} \cdot \mathrm{m}^{-2} \cdot \mathrm{nm} \mathrm{m}^{-1}$ and $L_{S}\left(\lambda ; \mathbf{x}_{S}, \boldsymbol{\omega}\right)$ has units $\mathrm{W} \cdot \mathrm{m}^{-2} \cdot \mathrm{sr}^{-1} \cdot \mathrm{nm}^{-1}$.

\section{The effective inverse surface function}

The light emitted by most practical lamps has the same spectral composition for all emission directions and all points of their emitting area. The source radiance $L_{S}\left(\lambda ; \mathbf{x}_{S}, \boldsymbol{\omega}\right)$ can therefore be factored out as the product of a spectral term $\Phi_{S}(\lambda)$ and a geometric one $B\left(\mathbf{x}_{\boldsymbol{S}}, \boldsymbol{\omega}\right)$ as:

$$
L_{S}\left(\lambda ; \mathbf{x}_{S}, \boldsymbol{\omega}\right)=\Phi_{S}(\lambda) B\left(\mathbf{x}_{S}, \boldsymbol{\omega}\right) .
$$


The factors $\Phi_{S}(\lambda)$ and $B\left(\mathbf{x}_{S}, \boldsymbol{\omega}\right)$ can be arbitrarily scaled, provided that their product gives the correct value for the source spectral radiance in equation (2). A convenient choice is to identify $\Phi_{S}(\lambda)$ with the spectral radiant flux of the source, measured in $\mathrm{W} \cdot \mathrm{nm}^{-1}$, and consequently expressing the remaining geometric factor, $B\left(\mathbf{x}_{S}, \boldsymbol{\omega}\right)$, in units $\mathrm{m}^{-2} \cdot \mathrm{sr}^{-1}$. Since the linear operator $\ell_{\lambda}$ acts on the source position and angular direction variables but not on the wavelength, after substituting equation (2) into equation (1) we get:

$$
E_{O}\left(\lambda ; \mathbf{x}_{O}, \mathbf{n}_{O}\right)=\Phi_{S}(\lambda) \cdot T\left(\lambda ; \mathbf{x}_{O}, \mathbf{n}_{O}\right)
$$

where

$$
T\left(\lambda ; \mathbf{x}_{O}, \mathbf{n}_{O}\right)=\ell_{\lambda}\left[B\left(\mathbf{x}_{S}, \boldsymbol{\omega}\right)\right]
$$

with units $\mathrm{m}^{-2}$, is the effective inverse surface function that, when multiplied by the source spectral radiant flux in $W \cdot n m^{-1}$, gives us the spectral irradiance in $W \cdot m^{-2} \cdot \mathrm{nm}^{-1}$ at the defined point.

\section{Discussion}

The effective inverse surface function, $T\left(\lambda ; \mathbf{x}_{O}, \mathbf{n}_{O}\right)$, describes the way in which the indoor environment modifies the original source spectrum to generate the spectral irradiance distribution at the defined point. In a loose sense $T\left(\lambda ; \mathbf{x}_{O}, \mathbf{n}_{O}\right)$ acts a filter that attenuates or enhances the different wavelengths as the light propagates from the source to the defined point. Note, however, that it is not a filter function in the proper sense of the term, since it relates two magnitudes with different units, and hence it is not dimensionless. 
The form of this function depends on the distribution of the source radiance, on the spectral reflectances of the environment, and on the position and orientation of the measurement surface, relative to the source and surfaces. Different kinds of lamps may therefore produce a slightly different functional form of $T\left(\lambda ; \mathbf{x}_{O}, \mathbf{n}_{O}\right)$, depending on their precise radiance distribution. However, this function is independent of the source spectrum. Once computed or measured for a given situation, it can be applied to any arbitrary lamp spectrum in order to predict the spectral irradiance at the observation point.

The effective inverse surface function can be numerically computed using equation (A7) if the radiance pattern of the source and the reflecting properties of the surrounding surfaces are known. This calculation involves a recursive series of integrals that, in practice, has to be truncated to a finite number of terms. This is a common situation in any real applications with multiple reflections. The number of terms that have to be included in the truncated series increases as the surface reflectance increases.

When the spectral reflectances of the surrounding surfaces are not precisely known, the effective inverse surface function can be determined experimentally, by measuring the spectral irradiance at the selected point of observation and dividing it by the spectral radiant flux of the source as:

$$
T\left(\lambda ; \mathbf{x}_{O}, \mathbf{n}_{O}\right)=E_{O}\left(\lambda ; \mathbf{x}_{O}, \mathbf{n}_{O}\right) \Phi_{S}(\lambda)
$$

Continuous spectrum sources should be used for this measurement, in order to avoid zeros or very low values in the denominator that would give rise to strong noise amplification and indeterminate values of $T$. 


\section{Funding}

This work was developed within the framework of the Spanish Network for Light Pollution Studies (Ministerio de Economía y Competitividad, grant AYA2015-71542REDT).

\section{References}

1. Bellia L, Pedace A, Fragliasso F. Indoor lighting quality: Effects of different wall colours. Lighting Research and Technology 1477153515594654, first published on July 10, 2015 as doi:10.1177/1477153515594654.

\section{Appendix}

Let us denote by $\mathbf{x}_{O}$ the position vector of a defined point located on an arbitrary surface within an indoor space, and by $\mathbf{n}_{O}$ the unit vector perpendicular to the surface at $\mathbf{x}_{O}$ (Figure 1). The spectral irradiance at that point, $E_{O}\left(\lambda ; \mathbf{x}_{O}, \mathbf{n}_{O}\right)$, is then:

$$
E_{O}\left(\lambda ; \mathbf{x}_{O}, \mathbf{n}_{O}\right)=\int_{\Omega_{S}} L_{S}\left(\lambda ; \mathbf{x}_{S}, \boldsymbol{\omega}_{O S}\right) \cos \alpha_{O S} \mathrm{~d} \omega_{O S}+\int_{\Omega_{e}} L_{e}\left(\lambda ; \mathbf{x}_{e}, \boldsymbol{\omega}_{O e}\right) \cos \alpha_{O e} \mathrm{~d} \omega_{O e}
$$

where the first integral represents the direct contribution of the light source, being $L_{S}\left(\lambda ; \mathbf{x}_{S}, \omega_{O S}\right)$ the spectral radiance of the source point $\mathbf{x}_{S}$ that arrives at the defined point from the direction of the unit vector $\boldsymbol{\omega}_{O S}=\left(\mathbf{x}_{S}-\mathbf{x}_{O}\right) /\left\|\mathbf{x}_{S}-\mathbf{x}_{O}\right\|$, with \|\| standing for modulus, $\alpha_{O S}=\arccos \left(\mathbf{n}_{O} \cdot \boldsymbol{\omega}_{O S}\right)$ is the angle between this direction and the normal $\mathbf{n}_{O}, \mathrm{~d} \omega_{O S}$ is an infinitesimal solid angle element around $\boldsymbol{\omega}_{O S}$, and the integral is 
extended to $\Omega_{S}$, the overall solid angle subtended by the source as seen from $\mathbf{x}_{O}$. The second integral represents the radiance reflected towards $\mathbf{x}_{O}$ from the points $\mathbf{x}_{e}$ of the environment, $L_{e}\left(\lambda ; \mathbf{x}_{e}, \omega_{O e}\right)$. The meanings of the magnitudes appearing therein $\left(\omega_{O e}, \alpha_{O e}, \mathrm{~d} \omega_{O e}\right.$ and $\left.\Omega_{e}\right)$ are analogous to the ones in the first integral, but now refer to the points on the surrounding surfaces instead of to the source. The solid angle domain $\Omega_{e}$ covers all the directions encompassed by the hemisphere above the plane that contains the defined point $(2 \pi$ steradians), excluding the points geometrically obstructed by the source. Henceforth the notation will follow the same conventions for the definition of the position vectors, unit vectors along the directions joining them, planar angles with respect to the normals to the surfaces, and solid angles.

The spectral radiance $L_{e}\left(\lambda ; \mathbf{x}_{e}, \omega_{O e}\right)$ directed to the defined point from any generic point $\mathbf{x}_{e}$ on the surfaces is given by the fraction of the light incident on that point that is diffusely reflected towards $\mathbf{x}_{O}$. Note that every surface point is illuminated not only by the primary light source located at $\mathbf{x}_{S}$ but also by the light reflected from the remaining unobstructed surface points $\mathbf{x}_{e^{\prime}} \neq \mathbf{x}_{e}$.

Let us denote by $\mathrm{d} E_{e}\left(\lambda ; \mathbf{x}_{e}, \boldsymbol{\omega}_{e e^{\prime}}\right)$ the elementary irradiance incident on $\mathbf{x}_{e}$ from an infinitesimal cone of directions $\mathrm{d} \omega_{e e^{\prime}}$ around the unit vector $\boldsymbol{\omega}_{e e^{\prime}}=\left(\mathbf{x}_{e^{\prime}}-\mathbf{x}_{e}\right) /\left\|\mathbf{x}_{e^{\prime}}-\mathbf{x}_{e}\right\|$. The elementary radiance reflected at $\mathbf{x}_{e}$ in the direction of the defined point, $d L_{e}\left(\lambda ; \mathbf{x}_{e}, \boldsymbol{\omega}_{O e}\right)$, can be related to $\mathrm{d} E_{e}\left(\lambda ; \mathbf{x}_{e}, \boldsymbol{\omega}_{e e^{\prime}}\right)$ by means of the space-variant bidirectional reflectance distribution function (svBDRF) $f\left(\lambda ; \mathbf{x}_{e}, \boldsymbol{\omega}_{o e}, \boldsymbol{\omega}_{e e^{\prime}}\right)$, defined as the ratio:

$$
f\left(\lambda ; \mathbf{x}_{e}, \boldsymbol{\omega}_{o e}, \boldsymbol{\omega}_{e e^{\prime}}\right) \equiv \mathrm{d} L_{e}\left(\lambda ; \mathbf{x}_{e}, \boldsymbol{\omega}_{O e}\right) / \mathrm{d} E_{e}\left(\lambda ; \mathbf{x}_{e}, \boldsymbol{\omega}_{e e^{\prime}}\right)
$$


But, by definition, $\mathrm{d} E_{e}\left(\lambda ; \mathbf{x}_{e}, \boldsymbol{\omega}_{e e^{\prime}}\right)=L_{e^{\prime}}\left(\lambda ; \mathbf{x}_{e^{\prime}}, \boldsymbol{\omega}_{e e^{\prime}}\right) \cos \alpha_{e e^{\prime}} \mathrm{d} \omega_{e e^{\prime}}$, where $L_{e^{\prime}}\left(\lambda ; \mathbf{x}_{e^{\prime}}, \boldsymbol{\omega}_{e e^{\prime}}\right)$ is the radiance of the surface point $\mathbf{x}_{e^{\prime}}$ in the direction of $\mathbf{x}_{e}$, $\alpha_{e e^{\prime}}=\arccos \left(\mathbf{n}_{e} \cdot \boldsymbol{\omega}_{e e^{\prime}}\right)$, and $\mathbf{n}_{e}$ is the unit vector normal to the surface at $\mathbf{x}_{e}$. Substituting this expression into equation (A2), solving for $\mathrm{d} L_{e}\left(\lambda ; \mathbf{x}_{e}, \boldsymbol{\omega}_{O e}\right)$, and integrating to the solid angle $\Omega_{e^{\prime}}$ subtended by all surface points seen from $\mathbf{x}_{e}$, we get the overall radiance reflected from $\mathbf{x}_{e}$ towards $\mathbf{x}_{O}$ due to the surrounding surfaces:

$$
L_{e, w a l l s}\left(\lambda ; \mathbf{x}_{e}, \boldsymbol{\omega}_{o e}\right)=\int_{\Omega_{e^{\prime}}} f\left(\lambda ; \mathbf{x}_{e}, \boldsymbol{\omega}_{O e}, \boldsymbol{\omega}_{e e^{\prime}}\right) L_{e^{\prime}}\left(\lambda ; \mathbf{x}_{e^{\prime}}, \boldsymbol{\omega}_{e e^{\prime}}\right) \cos \alpha_{e e^{\prime}} \mathrm{d} \omega_{e e^{\prime}}
$$

On the other hand, the light that arrives at $\mathbf{x}_{e}$ directly from the source contributes to the reflected radiance as:

$$
L_{e, \operatorname{source}}\left(\lambda ; \mathbf{x}_{e}, \boldsymbol{\omega}_{O e}\right)=\int_{\Omega_{S}} f\left(\lambda ; \mathbf{x}_{e}, \boldsymbol{\omega}_{O e}, \boldsymbol{\omega}_{e S}\right) L_{S}\left(\lambda ; \mathbf{x}_{S}, \boldsymbol{\omega}_{e S}\right) \cos \alpha_{e s} \mathrm{~d} \omega_{e s}
$$

The overall radiance directed from $\mathbf{x}_{e}$ towards $\mathbf{x}_{O}, L_{e}\left(\lambda ; \mathbf{x}_{e}, \omega_{O e}\right)$, which is the sum of the contributions of equations ( $A 3$ ) and ( $A 4)$, is then given by:

$$
\begin{array}{r}
L_{e}\left(\lambda ; \mathbf{x}_{e}, \boldsymbol{\omega}_{o e}\right)=\int_{\Omega_{S}} f\left(\lambda ; \mathbf{x}_{e}, \boldsymbol{\omega}_{o e}, \boldsymbol{\omega}_{e S}\right) L_{S}\left(\lambda ; \mathbf{x}_{S}, \boldsymbol{\omega}_{e s}\right) \cos \alpha_{e s} \mathrm{~d} \omega_{e s}+ \\
\int_{\Omega_{e^{\prime}}} f\left(\lambda ; \mathbf{x}_{e}, \boldsymbol{\omega}_{o e}, \boldsymbol{\omega}_{e e^{\prime}}\right) L_{e^{\prime}}\left(\lambda ; \mathbf{x}_{e^{\prime}}, \boldsymbol{\omega}_{e e^{\prime}}\right) \cos \alpha_{e e^{\prime}} \mathrm{d} \omega_{e e^{\prime}}
\end{array}
$$

Note that equation (A5) holds for any arbitrary surface point $\mathbf{x}_{e}$ and any direction $\omega_{O e}$. This equation expresses a self-consistent condition that shall be obeyed by the radiance of the environment, whereby the radiance reflected at any surface point depends on the radiance reflected at the remaining ones, as well as on the radiance 
received directly from the source. Substituting equation (A5) into equation (A1) we can write the spectral irradiance at the defined point as:

$$
\begin{aligned}
& E_{O}\left(\lambda ; \mathbf{x}_{O}, \mathbf{n}_{O}\right)=\int_{\Omega_{S}} L_{S}\left(\lambda ; \mathbf{x}_{S}, \boldsymbol{\omega}_{O S}\right) \cos \alpha_{O S} \mathrm{~d} \omega_{O S}+ \\
& \int_{\Omega_{e}}\left[\int_{\Omega_{S}} f\left(\lambda ; \mathbf{x}_{e}, \boldsymbol{\omega}_{O e}, \boldsymbol{\omega}_{e S}\right) L_{S}\left(\lambda ; \mathbf{x}_{S}, \boldsymbol{\omega}_{e S}\right) \cos \alpha_{e S} \mathrm{~d} \omega_{e S}\right] \cos \alpha_{O e} \mathrm{~d} \omega_{O e^{+}} \\
& \int_{\Omega_{e}}\left[\int_{\Omega_{e^{\prime}}} f\left(\lambda ; \mathbf{x}_{e}, \boldsymbol{\omega}_{O e}, \boldsymbol{\omega}_{e e^{\prime}}\right) L_{e^{\prime}}\left(\lambda ; \mathbf{x}_{e^{\prime}}, \boldsymbol{\omega}_{e e^{\prime}}\right) \cos \alpha_{e e^{\prime}} \mathrm{d} \omega_{e e^{\prime}}\right] \cos \alpha_{O e} \mathrm{~d} \omega_{O e}
\end{aligned}
$$

where the first term on the right-hand side corresponds to the light that arrives directly from the source to the defined point $\mathbf{x}_{O}$, the second corresponds to the light from the source that has undergone a single reflection at the surrounding surfaces, and the third is the overall contribution of the light that has undergone more than one reflection at the surfaces (i.e., the resultant of the reflection at every surface point of the diffuse light reflected from the remaining points).

The self-consistent relationship (equation A5) can be recursively substituted for the surface radiance $L_{e^{\prime}}\left(\lambda ; \mathbf{x}_{e^{\prime}}, \omega_{e e^{\prime}}\right)$ in the integrand of the last term of equation (A6) to rewrite this equation as a series of contributions of light scattered at the surfaces multiple times. Each term of this series corresponds to the light that underwent a given number of diffuse reflections at the walls before reaching $\mathbf{x}_{O}$. The explicit expression up to the double reflections is: 
$E_{O}\left(\lambda ; \mathbf{x}_{O}, \mathbf{n}_{O}\right)=\int_{\Omega_{S}} L_{S}\left(\lambda ; \mathbf{x}_{S}, \boldsymbol{\omega}_{O S}\right) \cos \alpha_{O S} \mathrm{~d} \omega_{O S}+$
$\int_{\Omega_{e}}\left[\int_{\Omega_{S}} f\left(\lambda ; \mathbf{x}_{e}, \boldsymbol{\omega}_{O e}, \boldsymbol{\omega}_{e S}\right) L_{S}\left(\lambda ; \mathbf{x}_{S}, \boldsymbol{\omega}_{e S}\right) \cos \alpha_{e S} \mathrm{~d} \omega_{e s}\right] \cos \alpha_{O e} \mathrm{~d} \omega_{O e}+$
$\int_{\Omega_{e}}\left[\int_{\Omega_{e^{\prime}}}\left[\int_{\Omega_{S}} f\left(\lambda ; \mathbf{x}_{e^{\prime}}, \boldsymbol{\omega}_{e e^{\prime}}, \boldsymbol{\omega}_{e^{\prime} S}\right) L_{S}\left(\lambda ; \mathbf{x}_{S}, \boldsymbol{\omega}_{e^{\prime} S}\right) \cos \alpha_{e^{\prime} S} \mathrm{~d} \omega_{e^{\prime} s}\right] f\left(\lambda ; \mathbf{x}_{e}, \boldsymbol{\omega}_{O e}, \boldsymbol{\omega}_{e e^{\prime}}\right) \cos \alpha_{e e^{\prime}} \mathrm{d} \omega_{e e^{\prime}}\right] \cos \alpha_{O e} \mathrm{~d} \omega_{O e}+\ldots$

Note that, as expected, the spectral irradiance at the defined point depends linearly on the spectral radiance of the source, $L_{S}\left(\lambda ; \mathbf{x}_{S}, \boldsymbol{\omega}\right)$ and, in a strongly nonlinear way, on the spectral reflectances of the surrounding surfaces. The linear dependence of $E_{O}\left(\lambda ; \mathbf{x}_{O}, \mathbf{n}_{O}\right)$ on $L_{S}\left(\lambda ; \mathbf{x}_{S}, \boldsymbol{\omega}\right)$ through the series (equation $\left.A 7\right)$ can be formally expressed as:

$$
E_{O}\left(\lambda ; \mathbf{x}_{O}, \mathbf{n}_{O}\right)=\ell_{\lambda}\left[L_{S}\left(\lambda ; \mathbf{x}_{S}, \boldsymbol{\omega}\right)\right]
$$

where $\ell_{\lambda}$ stands for linear operator. This is a wavelength-dependent operator acting on the spatial variables (position and orientation) through the integrals in equation (A7).

It is important to note that although all the equations presented here have been given in terms of spectral radiometry they also apply to spectral photometry. 